\title{
HIV Voluntary Counselling and Testing: A Qualitative Assessment of Teachers' Perceptions Regarding HIV status and VCT after an Intervention Workshop
}

\author{
Anthony-Williams Martin $\mathrm{H}^{\mathbf{1}^{*}}$, Anthony Williams Martin $\mathrm{A}^{2}$ and Kanu Hoisanatu $\mathrm{M}^{3}$ \\ ${ }^{1}$ Department of Microbiology, College of Medicine and Allied Health Sciences, University of Sierra Leone, \\ ${ }^{2}$ Famcare Medical Centre, Freetown, Sierra Leone, ${ }^{3}$ National HIV/AIDS Control Progamme, Ministry of \\ Health and Sanitation, Sierra Leone
}

\begin{abstract}
An intervention workshop was organised by a community-based project with aim to promote HIV Voluntary Counselling and Testing (VCT) and assess the perception of participants about the benefits of VCT and knowing their HIV status. The intervention workshop was conducted on a group of thirty teachers who consented to participate in the study. For the qualitative assessment, focus group discussions on three thematic topics were conducted and the outcome collated and analysed for consistency. The three themes were - benefits of an individual knowing his/her HIV status; harms of an individual knowing his /her HIV status; how to facilitate or encourage VCT uptake. The focal group discussions showed that there were fundamental psychosocial barriers to acceptability of VCT amongst the study participants. These included the issue of stigmatisation, fear of death from AIDS, negative effect on relationship and emotional depression. The study shows the need for healthcare professionals and policy makers involved in HIV/AIDS control to develop more patient- centred and friendly intervention that will overcome these negative perceptions of VCT.
\end{abstract}

\section{Keywords: HIV/AIDS, HIV status, Perception, Stigmatisation, VCT uptake}

Received 13 October 2009/ Accepted 4 December 2009

\section{INTRODUCTION}

The HIV/AIDS global pandemic has continued with a high prevalence especially in Sub-Saharan Africa and Asia. By the end of 2004, there were an estimated 42 million persons living with HIV/AIDS with 28.5 million were in Sub-Saharan Africa and 6 million in Asia (WHO, 2004). Further, by 2007 an additional 2.7 million new cases of HIV infection were reported with $67 \%$ of them occurring in Sub- Saharan Africa and Asia (UNAIDS, 2008). In Sierra Leone, a national population based sero-prevalence survey conducted in 2005 estimated a prevalence of $1.53 \%$ (NAS, 2005). The true prevalence could be much higher especially amongst high-risk groups such as commercial sex workers, intravenous drug users, the uniformed forces to mention a few. In addition to this, studies have shown that the age group most at risk of exposure to HIV infection are 15 to 49 years old (Mabey, 1990). A recent World Health Organisation (WHO) survey conducted by the African Regional Office (AFRO) reported HIV/AIDS prevalence amongst 15 to 49 years old as high as $23.6 \%$ (WHO, 2008). This is the prime working age, with most students and teachers in secondary schools within this age group.

The rate of uptake of Voluntary Counselling and Testing (VCT) in Sierra Leone is low, with an uptake rate of $6 \%$ amongst those included in the sero-prevalence survey conducted in 2005 (NAS, 2005). An increase in uptake rate of VCT will result in higher proportion of the population knowing their HIV status and thus adopting preventive measures if negative. 
Those who are positive will be advised to seek medical care and social support. This should lead to more effective prevention and control strategies of the HIV pandemic in the population. VCT is widely considered the cornerstone for HIV prevention in the control programme of many countries because of its numerous benefits (Bwanbale et al., 2008). However, the delivery of acceptable VCT services continues to be a challenge for HIV control programmes and other stakeholders.

The intervention workshop was conducted by a community-based project with aim to promote VCT and assess the perception of the participants about the benefits of VCT and knowing their HIV status. The target group were teachers of a secondary school in Freetown, Sierra Leone. The rationale for targeting teachers in this study is to give insight into how and why to change the perception of teachers to HIV test, as they form an important conduit for behavioural change and perception amongst adolescents (Kleep et al., 1997; Kawai et al., 2008).

\section{METHODOLOGY}

\section{Study setting and design}

The intervention workshop involved a group of thirty teachers who consented to participate in the workshop to improve their knowledge of HIV/AIDS and assess their perception of VCT for HIV and the merits to them of knowing their HIV status. The setting was a secondary school, located in the central part of Freetown, the capital of Sierra Leone. The teachers that participated in the study were randomly selected from their attendance register and each individual contacted for their consent. Six (6) teachers contacted refused to participate in the workshop, citing disbelief of HIV/AIDS and felt it was not a useful way to spend their weekends. The age of the participants ranged from 25 to 60 years, with a male/female ratio of 1.6. The workshop employed focus group discussions as the qualitative method of assessment of perception and the sample size was deemed appropriate for such a design.

The intervention at the workshop included presentations by three facilitators on the following topics: natural history and clinical stages of HIV/AIDS, mode of transmission, how and why to do VCT for HIV, HIV/AIDS preventive strategy in Sierra Leone. On the natural history and clinical stages of HIV/AIDS, participants were introduced to the World Health Organization clinical staging of HIV/AIDS and clinical manifestation of each stage. This was followed by a presentation on the common mode of transmission and what preventive strategies are employed in Sierra Leone. The common mode of transmission outlined included unprotected sexual intercourse with an infected partner (vaginal, anal or oral), exchange of blood and its products, from infected mother to child during childbirth or breast-feeding. The preventive strategy discussed were mainly primary preventive measures and included counseling (individual, couple and group), voluntary or provider initiated testing for HIV, use of information education and communication (IEC) materials, prevention of mother child transmission and free condom distribution. With regards to VCT, a demonstration of the testing procedure was done and the approach used by the National HIV/AIDS Control Programme (NACP), that is serial testing with the test kits; Determine (Abbot, Japan) $\rightarrow$ SD Bioline (SD Standard Diagnostic, Korea) $\rightarrow$ Unigold (Trinity Biotech, Ireland) (confirmatory). This was followed by a presentation on validity and safety VCT procedure and what care and social support facilities were available to persons who test positive for HIV.

\section{Data collection and analysis}

All participants were divided into three separate groups to discuss issues pertaining to each theme. Each group used a separate room to ensure their independence and a facilitator modulated the discussions. The discussions were centred about the three themes agreed upon by the researchers:

- benefits of an individual knowing his /her HIV status

- harms of an individual knowing his /her HIV status

- how to facilitate or encourage VCT uptake 
In each group a key person was selected who transcribed and presented the issues emerging from these themes. The facilitator's role was to ensure the discussion did not deviate from the themes, ensure that every member had an opportunity to express their opinions and the time (15 minutes) allotted to each theme was adhered to. There were no standard questions imposed on the group members. However, the group members had to reach a consensus on each issue raised in the group for it to be recorded and presented by the key person. Before the key person made his final presentation, his notes were read to group for final agreement. The weakness of this focus group discussion is that those members with a strong personality would have more influence on the discussions. In addition, there was the possibility of loss of content when summarising data. The data was synthesized by the inductive approach (Singleton et al., 1988) based on issues raised in discussions. The issues raised by each group were tabulated and those that were consistent amongst the three groups considered pertinent to that theme.

\section{RESULTS}

The issues raised by each focus group were tabulated for the three themes. Those issues that were agreed upon by each focus group were considered consistent with their perception to HIV and VCT (Figure 1). On the theme of benefit of VCT, the consistent issues were; it promotes good sexual practice in those found to have HIV negative status, it promotes Prevention of Mother to Child Transmission (PMTCT) and, it promotes medical care, support and positive living for People Living with HIV and AIDS (PLWHA). With regards the theme of harms of VCT, the consistent issues were; the fear of death from AIDS, stigmatisation and its impact on relationships, psychological depression and selfcondemnation. On the theme of how to encourage VCT uptake, the consistent issues were; the improvement of health education on HIV/AIDS and VCT, increase availability of free testing and ensure confidentiality.

\section{DISCUSSION}

The traditional approach of VCT with emphasis on pre-test counselling has shown some prospects in promoting sexual behaviour change and decreasing risk of sexual transmission (Sweat et al., 2000). On the contrary, the availability and rate of uptake of VCT has continued to be very low, particularly in populations where the prevalence of HIV is high (UNAIDS, 2001). This paradox is one of the greatest challenges to HIV prevention and control programmes. This study attempted to combine an intervention designed to improve the knowledge of participants on HIV and VCT and assess their perception to VCT. A study by Kawai et al (2008) showed an important role of teachers' knowledge of HIV and communication in influencing adolescents' perception to safe sexual practice.

The focus group discussions, a qualitative methodology, showed that there were fundamental psychosocial barriers to acceptability of VCT amongst the study participants. These included the issue of stigmatisation, fear of death from AIDS, negative effect on relationship and emotional depression. A study by lliyasu et al. (2006) on knowledge of HIV/AIDS and Attitude to VCT reported similar findings wherein fear of stigma, marital disharmony and incurable nature of HIV/AIDS and cost of treatment were the main reasons for rejection of VCT. Concerning the benefit of VCT and how to encourage VCT uptake, the issue of good sexual practice, prevention of mother to child transmission, medical care and support for PLWHA were the main benefits, whilst improved health education on HIV/AIDS and VCT, increased availability and confidentiality were crucial to improved uptake. These are consistent with a study by Wringe et al. (2008), in which they highlighted certain predictor factors such as perceived risk of HIV infection, prior knowledge of VCT influenced uptake but that interventions to improve knowledge of HIV risk, and benefit of VCT should be tailored to clients.

In a recent Cochrane systematic review that critically appraised studies on different approach to implementation of VCT showed that home base testing and delivery of results at home lead to much higher VCT uptake than facility based testing (Bateganye et al., 2007). The study methodologies included in the review varied from before/after studies, cluster randomised trials and randomised control trials. This provides further evidence to support the argument that VCT intervention be evaluated at community level and the innovative approaches adopted to improve uptake. 
This study had the following limitations; a small sample of participants who were more enlightened or educated compared with the general population. The selection of the study sample was quasi randomised with some bias as only those who readily volunteered participated in the workshop. This might have excluded those with a negative perception of HIV/AIDS or VCT. It may not be representative of teachers, as only those in one school were surveyed. Furthermore, the strength of such a study and the inherent weaknesses of qualitative analysis will cause some controversy in the scientific world. Studies related to HIV testing and VCT uptake highlighted in discussion above were found in the search of literature via Pubmed, EMBASE database, Cochrane reviews and relevant professional website. However, to the best of our knowledge, there were no documented studies on the perception of teachers in relation to their HIV status and acceptance of VCT.

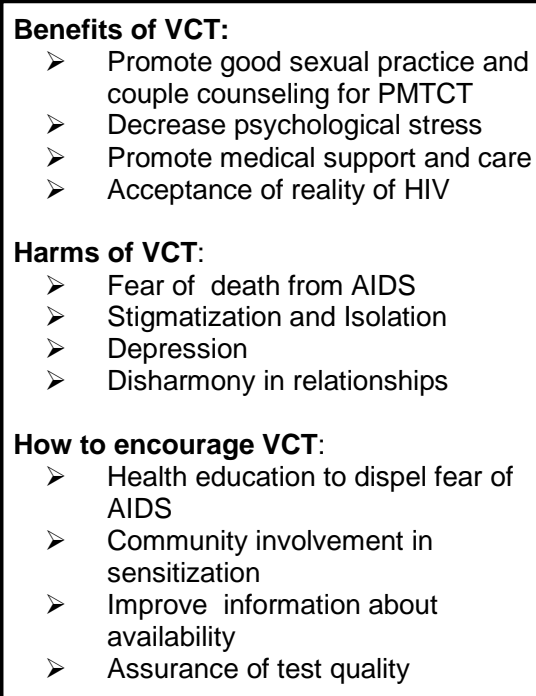

$>$ Promote good sexual practice and couple counseling for PMTCT

$>$ Decrease psychological stress

$>$ Promote medical support and care

$>$ Acceptance of reality of HIV

Harms of VCT:

$>$ Fear of death from AIDS

> Stigmatization and Isolation

$>$ Depression

$>$ Disharmony in relationships

How to encourage VCT:

$>$ Health education to dispel fear of AIDS

$>$ Community involvement in sensitization

> Improve information about availability

$>$ Assurance of test quality

Summary of Issues

Consistent in Each Focus Group

\section{Benefit of VCT:}

$>$ Promote PMTCT

$>$ Promote good sexual behaviour

$>$ Promote VCT to others

> Medical care and social support for positive living

Harms of VCT:

$>$ Fear of death ,psychological depression

$>\quad$ Lack of confidentiality

> Deliberate spread likely

> Breakdown in relationship

\section{How to encourage VCT:}

$>$ Health education on VCT

$>$ Well trained VCT staff

$>$ Assured free test and confidentiality $>$ Increase VCT sites
Benefit of VCT

$>$ Promotes PMTCT

$>$ Ensure proper treatment for infected persons and social support

> Promote good sexual practice and behaviour

Harms of VCT:

$>\quad$ Lack of cure and fear of death

$>$ Self condemnation

$>$ Stigmatization

> Relationship breakdown

How to encourage VCT:

$>$ Compulsory testing before entry to tertiary institutions

$>$ Health education to dispel fears of AIDS

$>$ Ensure free testing and treatment

$>$ Educate students of risk of HIV infection

Figure 1: Outcome of Focus Group Discussions 


\section{CONCLUSIONS}

The qualitative study shows that there is need for health professionals involved in HIV/AIDS prevention and control to develop more interventions that are patient centred and friendly that will help overcome the negative perceptions of VCT. Studies that focus on understanding the client or patients perceptions and interventions that improve their knowledge of HIV/AIDS and the merits of VCT have demonstrated a positive impact on VCT uptake and hence HIV/AIDS prevention and control. Approaches of a provider initiated rather than client initiated counselling and testing, or home-based rather than facility-based VCT may improve on uptake, but these must be evaluated periodically, not only from a quantitative perspective but also psychosocial. This will empower health professional involved in HIV/AIDS prevention and control and policy makers to identify barriers that limit the acceptability of VCT and develop strategies to overcome them and hence have a significant influence on policy formulation, planning and implementation of interventions to enhance VCT. The study showed that there were negative perceptions to HIV testing amongst the participants which need to change as well as positive ones that should be re-enforced. Such qualitative studies are a useful approach to understanding and facilitating change of perception to HIV and VCT. This small single study is however inadequate to affect policy and practice, thus more population wide studies are needed to impact policy decision and practice.

\section{ACKNOWLEDGEMENT}

Many thanks to the principal and staff, especially the health counsellor for their support in getting participants for the workshop. We are also grateful to the director and staff members of National HIV/AIDS Secretariat through which the funds for the Famcare-SHARP project were accessed.

\section{REFERNCES}

Bategenya M.H, Abdulwadud OA and Kiene SM (2007). Home- based HIV voluntary counseling and testing in developing countries. Cochrane Database Systematic Rev. 4: CD006493
Bwanbale FM, Ssali SN, Byaruhuhanya S, Kalyango JN and Karamagi C (2008). Voluntary HIV counseling and testing among men in rural western Uganda: Implications for HIV prevention. BMC Public Health. 8:263. Available from http://www.biomedcentral.com/1471-2458/8/263 [Accessed on 5 November 2008]

Iliyasu Z, Abubakar IS, Kabir M and Aliyu MH (2006). Knowledge of HIV/AIDS and Attitude towards Voluntary Counseling and Testing amongst Adults. J National Med Assoc. 98 (12): 1917-22

Kawai K, Kaaya SF, Kajula L, Mbwambo J, Kilonzo GP, Fawzi WW (2008). Parents' and Teachers' Communication about HIV and Sex in Relation to the Timing of Sexual Initiation among Young Adolescents in Tanzania. Scandinavian $J$ Public Health. 36 (8): 879-88

Kleep KI, Ndeki SS, Leshabari MT, Hannan PJ and Lyima BA (1997). AIDS Education in Tanzania: Promoting Risk Reduction Among Primary School Children. American J Public Health. 87(12):1932-6

Mabey D (1990). Sexual Transmitted Disease in Developing Countries. Reproductive Health J. 2: 96-100

NAS (2005). National Population Based HIV Sero-prevalence Survey of Sierra Leone. National HIV/AIDS Secretariat. Ministry of Health and Sanitation, Sierra Leone.

Singleton $R$, Straits $B$, Straits $M$ and McAllister $R$ (1988). Approaches to Social Research. Oxford: Oxford University Press. Pp: 295 -299.

Sweat M, Gregorich S, Sangiwa G, Furlonge C, Balmer D, Kamenga C, Grinstead O and Coates T (2000). Cost-effectiveness of Voluntary HIV-1 Counselling and Testing in Reducing Sexual Transmission of HIV-1 in Kenya and Tanzania. Lancet. 356 (9224):113 - 121 
UNAIDS (2001). The Impact of Voluntary Counseling and Testing: A global Review of the Benefits and Challenges. Joint United Nations Programme on HIVIAIDS (UNAIDS). ISBN 929273-077-7

UNAIDS (2008). Report on the Global AIDS Epidemic. Joint United Nations Programme on HIV/AIDS. UNAIDS/08.25E/JC151OE

WHO (2004). TB/HIV: A Clinical Manual World Health Organization. $2^{\text {nd }}$ Ed.

WHO/HTM/TB/2004.329
WHO (2008). HIV/AIDS Epidemiological Surveillance Report for WHO African Region Update. WHO. AFRO.

Wringe A, Isingo R, Urassa M, Maiseli G, Manyalla R, Changalucha J, Mngara J, Kalluvya $S$ and Zaba B (2008). Uptake of HIV Voluntary Counselling and Testing Services in Rural Tanzania: Implications for Effective HIV Prevention and Equitable Access to Treatment. Tropical Med Intern Health. 13 (3): 319-27 